\title{
Le cerveau humain fabrique-t-il ses propres tranquillisants?
}

Est-il encore besoin de présenter les benzodiazépines? Ces médicaments, très largement prescrits depuis plus de 30 ans, sont utilisés principalement comme sédatifs et anxiolytiques. Voici près de 15 ans, un récepteur de ccs molécules était isolé dans le cerveau humain, au niveau des membranes de cellules nerveuses [1, 2]. Depuis, l'identification d'une molécule synthétisée par l'organisme et active sur le récepteur dans des conditions physiologiques ou pathologiques - appelée ligand endogène du récepteur demeure toujours un sujet de controverse.

Deux récents travaux viennent de relancer le débat de l'existence de ce ligand : l'un [3], d'Anthony S. Basile, de l'Université de Bethesda (Maryland, MD, USA), implique la présence d'un ligand cndogène dans la genèse d'une affection neurologique, l'encéphalopathie hépatique, résultant d'une attcinte hépatique grave; l'autre [4], de l'équipe d'Ivan Izquierdo, du Centre de recherche sur la biochimie de la mémoire à Porto Allegre (Brésil), interprète une amélioration des performances mnésiques également par l'intervention d'un ligand endogène des réccpteurs des benzodiazépines. Avant d'entrer plus avant dans le débat, et de voir comment un éventuel ligand endogène du récepteur des benzodiazépines pourrait accomplir des tâches aussi variées, rappelons que ce récepteur fait partie d'un " complexe supramoléculaire " subdivisé en un site récepteur pour le GABA (acide $\boldsymbol{\gamma}$ amino-butyrique), un site récepteur pour les benzodiazépines et un canal ionique au chlore, qui porte lui-même des sites de reconnaissance pour les barbituriques, les stéroïdes et des molécules convulsivantes comme la picrotoxine. Elisabeth Bacon et Françoise Viennot ont présenté unc revuc détaillée de ce complexe récepteur GABAer-
La fonction du récepteur des benzodiazépines est de moduler l'ouvcrture du canal chlorure en présence de GABA, principal neuromédiateur inhibiteur du système nerveux central. On parle de modulation "allostérique ", puisque cette modulation s'effectue par des changements de conformation spatiale des récepteurs en présence de leur ligand spécifique. L'occupation du récepteur des benzodiazépines par un agoniste, par exemple le diazépam (Valium $\left.{ }^{\circledR}\right)$, ou toute molécule mimant son action, augmente l'affinité du GABA pour son propre récepteur et accroît la fréquence d'ouverture du canal chlore. Les principales propriétés pharmacologiques des benzodiazépines (effet sédatif, anxiolytique, anticonvulsivant et amnésiant), mais aussi d'autres agonistes non-benzodiazépiniques s'effectuent par l'intermédiaire de ce mécanisme. A l'opposé, des " agonistes inverses " des récepteurs des benzodiazépines (comme la $\beta$ CCM ou la DMCM appartenant à la famille chimique des $\beta$-carbolines) possèdent des propriétés convulsivantes, anxiogènes et promnésiantes, dues à leur capacité de diminuer l'affinité du GABA pour son récepteur. Enfin, il existe des antagonistes des récepteurs des benzodiazépines, comme le flumazénil (Anexate ${ }^{\circledR}$ ) qui ne modifient pas l'affinité du récepteur pour le GABA mais entrent en compétition avec les agonistes et les agonistes inverses au niveau des sites de liaison sur les récepteurs des benzodiazépines et bloquent leurs effets respectifs. Quel que soit le type de ligand, il ne modifie pas, par lui-même, la conductance au chlore, la présence de GABA est indispensable pour qu'une action puisse se manifester.

Il existe donc au sein du complexe récepteur GABA/benzodiazépines/canal chlore un site "effecteur ", le site de fixation du GABA, normalement activé par son ligand naturel pour entraîner une inhibition neuronale, et un site " modulateur ", le site des benzodiazépines, ainsi dénommé car historiquement les benzodiazépines ont été les premières molécules connues à s'y lier. Cependant, comme c'est le cas avec les $\beta$-carbolines, il existe d'autres familles chimiques qui peuvent se fixer spécifiquement sur le récepteur des benzodiazépines, et au sein desquelles se retrouvent les trois types fonctionnels de ligands : agonistes, agonistes inverses et antagonistes.

Ainsi, on a rapidement disposé d'un très grand nombre de molécules "exogènes " capables de se lier aux sites des benzodiazépines, et présentant des profils pharmacologiques variés, voirc opposés. La question restait de savoir s'il existait un ligand "endogène ", synthétisé par l'organisme humain, capable de se fixer spécifiquement sur le récepteur des benzodiazépines et de moduler l'action du GABA. De même que la mise en évidence de récepteurs morphiniques dans le cerveau avait été le prélude à la découverte de substances morphiniques intra-cérébrales, les enképhalines et les endorphines [7], par analogie on chercha très activement à isoler des substances susceptibles d'être des ligands endogènes des récepteurs des benzodiazépines, dès la découverte de ceux-ci. La liste des candidats proposés à cette fonction depuis 15 ans est longue. Nombreux sont ceux qui ont été éliminés du fait de leur faible spécificité : bien que reproduisant la plupart des actions des benzodiazépines, ils devaient être utilisés à des doses trop fortes non compatibles avec unc action physiologique. D'autres, comme les $\beta$-carbolines, un moment pressenties, sont apparues être des molécules artéfactuelles, néoformées au cours des processus d'extraction chimique à partir d'extraits biologiques. Actuellement, deux grandes hypothèses dans la recherche du ligand endogène des récepteurs des benzodiazépines coexistent : 
- l'une, soutenue par les travaux évoqués au début de cet article, défend l'existence d'un ligand endogène de type agoniste, possédant donc une action sédative, anxiolytique et perturbatrice de la mémoire ;

- l'autre, fondée sur les travaux de Costa et Guidotti [8], du Fidia Georgetown Institute, de Washington, argumente en faveur d'un ligand endogène de type agoniste inverse, c'est-à-dire anxiogène et facilitateur de la mémoire. En faveur de l'hypothèse d'un ligand de type agoniste, sédatif, des conclusions concordantes sont rapportées par deux séries de travaux très différents : A.S. Basile [3], ainsi que d'autres auteurs, plaide en faveur d'un ligand de type benzodiazépine à partir de l'étude de la genèse et du traitement de l'encéphalopathie hépatique. Ce syndrome neuro-psychiatrique se caractérise par une altération de la conscience plus ou moins profonde, pouvant aller jusqu'au coma, associéc à des troubles du tonus musculaire. Il résulte d'une dégradation importante de la fonction hépatique, pouvant apparaître au décours d'une maladie aiguë (hépatite virale ou toxique) ou chronique (cirrhose) du foie. Basile rapporte la présence de taux élevés de benzodiazépines "endogènes ", bien supérieurs à ceux que donneraient une administration à doses thérapeutiques de benzodiazépines, dans le ccrveau de patients décédés, atteints d'encéphalopathie hépatique, et n'ayant jamais reçu auparavant de traitement à base de ces médicaments. Ces importants résultats s'ajoutent à des données antérieures qui montraient une spectaculaire amélioration de l'état de malades en coma hépatique après administration de flumazénil, l'antagoniste du récepteur des benzodiazépines.

Une hypothèse vient relier ces éléments : l'encéphalopathie hépatique s'accompagnerait d'une élévation importante de benzodiazépines endogènes, elles-mêmes responsables des troubles de conscience (sédation massive). L'administration de flumazénil réduirait voire supprimerait l'action de ces molécules, conduisant à l'amélioration de l'état clinique observée. L'hypothèse est séduisante, mais plusieurs questions se posent à ce stade : d'où viennent ces benzodiazépines endogènes ? Ontelles un rôle physiologique ? Lequel ?
Les réponses apportées à la suite de ces découvertes sont encore incomplètes.

- L'origine de ces benzodiazépines endogènes est actuellement inconnue : il ne semble pas que l'homme, ni même l'ensemble des mammifères, sachent synthétiser le noyau chloré qui constitue le squelette chimique des benzodiazépines. La présence de $\mathrm{N}$ desméthyldiazépam, métabolite commun à plusieurs benzodiazépines, et de diazépam - synthétisé et commercialisé par les Laboratoires Roche sous le nom de Valium $^{\circledR}$ - a été démontrée dans divers tissus tant chez l'animal que chez l'homme (rate, foie, rein, cerveau et plasma). Une contamination lors des processus d'extraction a rigoureusement été exclue. Par ailleurs, ces dérivés ont été retrouvés dans du tissu cérébral de sujets décédés bien avant la mise sur le marché des benzodiazépines. Malgré ces résultats, il est peu probable que des dérivés de type benzodiazépine soient synthétisés de novo par les mammifères, la voie de synthèse enzymatique n'ayant pas étć identifiée. Un seul argument indirect d'une synthèse possible de tels dérivés par des cellules eucaryotes est disponible actuellement : ils ont été identifiés dans les milieux de culture de certaines cellules tumorales.

- Une alternative possible pour expliquer la présence de dérivés benzodiazépines dans l'organisme humain rćside dans le fait que leur synthèse est connue chez des micro-organismes microbiens et des champignons [9]. La présence de benzodiazépines endogènes chez l'homme pourrait donc provenir de deux sources " externes " non exclusives : soit une synthèse par la flore microbienne présente dans la lumière intestinale, soit un apport alimentaire. Cette deuxième hypothèse est plus documentée: de nombreux auteurs rapportent en effet la présence à l'état de traces de dérivés chimiques de type benzodiazépine dans un grand nombre d'aliments appartenant à la ration alimentaire humaine : le blé, la pomme de terre, le riz, le maïs, le soja, les lentilles et les champignons. Les végétaux sont donc capables d'assurer la synthèse de dérivés de type benzodiazépine, dont le desméthyldiazépam et le diazépam. Les concentrations trouvées sont très faibles, de l'ordre de quelques fractions de nanogrammes $\left(10^{-9}\right)$ par gramme d'aliment. Cependant, elles peuvent peut-être suffire à expliquer les taux également très faibles de ces produits retrouvés dans le tissu cérćbral de nombreux animaux et le plasma de sujets volontaires sains indemmes de prise antérieure de benzodiazépines. Récemment, ces mêmes benzodiazépines ont été isolées dans le lait maternel. Une contamination par pollution chimique massive, d'origine industrielle, transmise à l'homme par la chaînc alimentaire, avait un temps été envisagće. Mais elle a été exclue quand on a retrouvé la présence de dérivés de typc benzodiazépine dans les cerveaux de sujets décédés avant la disponibilité sur le marché de ces produits. L'apport alimentaire d'origine végétale semble donc être le plus plausible.

Si un apport alimentaire peut être légitimement envisagé, il faut souligner qu'il conduit à des doses de produit actif infimes au niveau du cerveau humain, incapables d'entraîner des cffets chimiques en conditions normales. Si l'on se réfère aux dosages cffectués chez l'homme et l'animal, il est même probable qu'aucune activité biologique ne puisse être attendue des concentrations trouvées. Doit-on continuer de considérer ces benzodiazépines alimentaires comme des candidats sérieux pour une liaison avec les récepteurs concernćs dans les conditions physiologiques ? Pcut-êtrc faut-il envisager une répartition intra-cérébrale hétérogène, compatible avec unc action neuromodulatrice ponctuelle de ce ligand au sein de structures spécialisées. Dans le cas de l'encéphalopathie hépatique, les quantités de dérivés de type benzodiazépine trouvés sont considérables, compatibles avec un effet clinique. Pour en expliquer l'origine, il faudrait envisager une accumulation de ces produits, par exemple par défaut de catabolisme, le foie lésé ayant perdu sa fonction d'épuration. Une forte élévation du taux de benzodiazépines endogènes jointe à l'accumulation d'autres produits neurotoxiques non dégradés par le foie serait à l'origine des troubles de conscience observés en clinique.

Ivan Izquierdo [4], pour sa part, défend l'hypothèse d'un ligand endogène, également de type benzodiazé- 
pine, en se fondant sur des résultats d'études comportementales chez l'animal : à partir d'expériences d'apprentissage et de mémorisation, il montre que la consolidation de la mémoire après un apprentissage simple est réglée de façon inhibitrice par un mécanisme GABAergique, lui-même modulé par un ou plusieurs ligands endogènes de type benzodiazépine libérés pendant l'apprentissage.

Pour comprendre un tel mécanisme d'action, il est nécessaire de se reporter aux effets des benzodiazépines observés en thérapeutique. La perturbation des apprentissages par la prise de benzodiazépines - action " amnésiante" - est un effet secondaire connu des cliniciens. De nombreuses observations rapportent une incapacité à apprendre, un " oubli à mesure ", contemporain de la prise de ces médicaments. Parallèlement, il a été montré que les $\beta$-carbolines, agonistes inverses, avaient la faculté d'améliorer les performances dans différentes tâches d'apprentissage chez l'animal [10]. Mais l'effet le plus inattendu revient au flumazenil: cet antagoniste ne devrait en théorie rien provoquer quand il est administré seul. Or, plusieurs auteurs, dont Izquierdo, rapportent une amélioration des performances d'apprentissage sous flumazénil [11, 12]. De tels résultats ayant été obtenus avec de faibles doses du produit, une action propre du flumazénil peut être exclue. Il est donc tentant d'envisager l'existence d'un ligand endogène libéré pendant l'apprentissage. Comme le blocage de ce ligand s'accompagne d'une amélioration des performances, c'est qu'il est de type benzodiazépine. Toutes les tâches d'apprentissage ne sont pas églement modifiées par le flumazénil. Izquierdo constate que les tâches les plus sensibles sont celles comportant un certain degré de stress pendant l'apprentissage. Le flumazénil est d'autant plus actif qu'il est utilisé dans le cadre d'un apprentissage plus anxiogène pour l'animal.

D'autres arguments sont venus corroborer ces résultats : il existe une diminution des possibilités de liaison de molécules radioactives sur les récepteurs des benzodiazépines cérébraux après certains apprentissages chez l'animal. Cela suggère qu'une occupation des récepteurs a pu se faire par la libé- ration d'un ligand interne pendant l'apprentissage. D'autres travaux montrent que l'intensité de la diminution de la liaison de molécules radioactives est fonction du type d'apprentissage : elle est beaucoup plus forte pour des tâches faisant intervenir un certain degré d'anxiété

Il existe ainsi de sérieux arguments pour penser que la première étape de la mémorisation d'une tâche d'apprentissage est sous la dépendance d'un mécanisme inhibiteur GABAergique, lui-même réglé par un ligand endogène de type benzodiazépine. La libération de ce ligand se ferait en proportion variable, fonction de l'intensité du stress engendré par le type d'apprentissage utilisé. L'acquisition d'une information nouvelle déclencherait un contrôle inhibiteur sur les processus de mémorisation. Celui-ci serait d'autant plus renforcé initialement par un ligand endogène de type benzodiazépine que les conditions d'acquisition seraient anxiogènes. Voilà donc, défini par Izquierdo, un rôle possible de ce ligand ; mais son origine est tout aussi indéterminée que précćdemment dans l'encéphalopathie hépatique. Et les mêmes hypothèses sont formulées. $\mathrm{La}$ question reste de savoir si les benzodiazépines trouvées au niveau cérébral, d'origine probablement alimentaire, peuvent être pressenties comme des régulateurs physiologiques de processus d'apprentissage.

$\mathrm{Si}$ les résultats qui viennent d'être exposés jusqu'ici sont convaincants, les travaux concernant l'existence possible d'un ligand endogène ayant des effets opposés à ceux des benzodiazépines, et donc de type agoniste inverse, anxiogène et facilitateur de la mémoire, ne sont pas moins argumentés. Le diazepam binding inhibitor (DBI) (inhibiteur de la liaison du diazépam) a été mis en évidence dans le cerveau humain en 1986. C'est un peptide de 104 acides aminés dont le gène est porté par le bras court du chromosome 2. Plusieurs fragments, supposés être les formes actives, ont été isolés. Il existerait des segments à activité agoniste inverse, et d'autres à activité agoniste. Ces fragments n'ont pas encore été séquencés chez l'homme.

Le DBI se fixe au niveau des sites des benzodiazépines, mais certains éléments s'opposent à faire de lui le ligand électif de ces récepteurs :

- son action sur la transmission GABAergique nécessite des concentrations macromolaires, 100 à 1000 fois supérieures aux concentrations habituellement nécessaires pour assurer une reconnaissance hautement spécifique entre ligand et récepteur ;

- le DBI est également présent dans des neurones non-GABAergiques, dans des cellules gliales, et également dans des organes périphériques tels que le foie, les surrénales, les organes génitaux, au niveau desquels il assure une régulation de la synthèse des hormones stéroïdes.

Ainsi, comme beaucoup d'autres neuropeptides, le DBI est présent dans de nombreuses structures du système nerveux et dans des organes périphériques, où il semble qu'on puisse lui attribuer plusieurs rôles physiologiques. Sa présence comme cotransmetteur n'est pas indispensable à la transmission GABAergique, puisqu'il n'est colocalisé avec le GABA dans des terminaisons nerveuses que dans la proportion d'une fois sur deux. On ne peut donc pas véritablement le considérer comme le ligand endogène essentiel à la régulation de la fonction inhibitrice du GABA, même si divers arguments peuvent suggérer un rôle possible de cette molécule dans le déterminisme de l'anxiété.

En conclusion, depuis plusieurs années que le récepteur des benzodiazépines a été identifié, l'existence d'un ou plusieurs ligands endogènes actifs dans des conditions normales ou pathologiques n'est pas encore une certitude. Au-delà du débat sur la nature de ce ligand, Willy Haefely, des Laboratoires Hoffman-Laroche (Bâle, Suisse), pose la question de la nécessité d'un ligand endogène pour les récepteurs des benzodiazépines [13]. Pour beaucoup la réponse est oui. En effet, pourquoi l'évolution des espèces aurait-elle maintenu pendant des millions d'années la présence d'un site modulateur de l'action du GABA s'il n'y avait pas de ligand endogène ? Il est difficilement envisageable qu'une protéine réceptrice soit conservée, capable de répondre à la fixation de molécules exogènes, s'il n'y avait pas de molécules endogènes jouant le rôle de modulateur physiologique. Cependant, le but essentiel était 
de garder fonctionnel un système inhibiteur efficace, et donc de préscrver lc récepteur du GABA. Pour Haefely, c'est lc récepteur effecteur qui est primordial, la modulation de l'activité de cclui-ci étant moins essentielle, voire pcut-être même pas nécessaire. L'hypothèse qu'un récepteur muni d'un canal ionique ne pourrait pas porter de sites à haute affinité pour des substances exogènes s'il n'existe pas de ligand endogène n'est, selon lui, pas fondée. Un argument avancé par Haefcly pour l'illustrer est que les immunoglobulines, protéines fabriquées par l'organisme, sont électivement destinées à la reconnaissance de molécules exogènes.

Concernant le réceptcur du GABA, il est concevable que cette volumineuse protéinc comporte sur sa surface un nombre considérable de groupements fonctionnels au niveau desquels de très petits composés exogènes viennent se fixer du fait de leur taille, leur forme et lcur charge électrique complémentaires. Il n'est pas improbable que parmi ces nombreux composés, certains se fixent avec une haute affinité, ct que parmi eux, un petit nombre puisse entraîner un changement conformationnel dans la structure protéique d'amplitude suffisante et au niveau d'un site physiologiquement fonctionnel. La probabilité d'une telle éventualité est certainement faible mais n'est pas nulle. Les protéines sont des moléculcs flexibles, en continuelle oscillation entre diverses conformations. Provoquer ou bloquer de tels mouvements cntre des conformations est la condition nécessaire pour qu'un ligand quelconque devienne un ligand actif ou un médicament. Il semblerait donc que la misc cn évidence d'un site modulateur ne soit pas une preuve a priori de l'existence d'un ligand endogène

\section{RÉFÉRENCES}

1. Braestrup C, Squires RF. Brain spccific benzodiazepine receptors. BrJ Psychiat 1978 ; 133 : 249-60.

2. Mohler H, Okada T. The benzodiazepine receptor in normal and pathological human brain. Br J Psychiat 1978; 133 : 261-9.

3. Basile AS. The contribution of endogenous benzodiazepine receptor ligands to the pathogenesis of hepatic encephalopathy. Synapse $1991 ; 7: 141-50$.

4. Izquierdo I, Pereira ME, Medina JH. Benzodiazepine receptor ligand influences on acquisition : suggestion of an endogenous modulatory mechanism mediated by benzodiazepine receptors. Behav Neurol Biol 1990 ; 54 : 27-41.

5. Bacon $\mathrm{E}$, Vicnnot $\mathrm{F}$. Le système complexe des récepteurs GABA-benzodiazépines. médecine/sciences $1990 ; 6: 770-7$

6. Olsen RW, Tobin AS. Molecular biology of GABA A receptors. FASEB J 1990; 4: 1469-80.

7. Rossier J, Chapouthier G. Enképhalines et endorphines. La Recherche 1982; 138: 1296-306.

8. Costa E, Guidotti A. Diazcpam binding inhibitor: a peptide with multiple biological actions. Life Sci 1991; 49 : 325-44.

9. Klotz U. Occurence of "natural " benzodiazepines. Life Sci 1991; 48 : 209-15

10. Chapouthier G. Des molécules pour la mémoire. La Recherche $1987 ; 192$ : 1258-60.

11. Lal H, Kumar B, Forster MJ. Enhancement of learning and memory in mice by a benzodiazepine antagonist. FASEB J 1988 ; 2 : $2707-11$

12. Raffalli-Scbille MJ, Chapouthier G. Similar effects of a $\beta$-carboline and of flumazenil in negatively and positively reinforced lcarning tasks in micc. Life Sci 1991; 48 : 685-92.

13. Haefely W. Endogenous ligands of the benzodiazepine receptor. Pharmacopsychiatry $1988 ; 21: 43-6$

\section{TIRÉS A PART}

Marie J. Raffalli-Sebille

Georges Chapouthier

Laboratoire de génétique, neurogénétique et comportement, URA 1294 CNRS, UFR biomédicale, université Paris $V, 45$, nue des Saints-Pères 75270 Paris Cedex 06, France. 